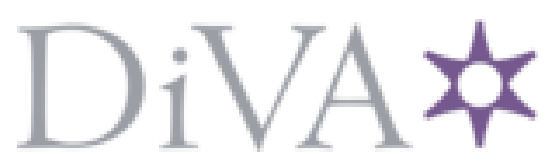

http://www.diva-portal.org

This is the published version of a paper published in Applied Physics Letters.

Citation for the original published paper (version of record):

Pettersson, H., Bååth, L., Carlsson, N., Seifert, W., Samuelson, L. (2001)

Case study of an InAs quantum dot memory: Optical storing and deletion of charge.

Applied Physics Letters, 79(1): 78-80

http://dx.doi.org/10.1063/1.1382628

Access to the published version may require subscription.

N.B. When citing this work, cite the original published paper.

Copyright of Applied Physics Letters is the property of American Institute of Physics and its content may not be copied or emailed to multiple sites or posted to a listserv without the copyright holder's express written permission. However, users may print, download, or email articles for individual use.

Permanent link to this version:

http://urn.kb.se/resolve?urn=urn:nbn:se:hh:diva- 1840 


\title{
Case study of an InAs quantum dot memory: Optical storing and deletion of charge
}

\author{
H. Pettersson ${ }^{\text {a) }}$ and L. Bäàth \\ Electronics and Physics Laboratory, Halmstad University, Box 823, SE-301 18 Halmstad, Sweden \\ N. Carlsson, W. Seifert, and L. Samuelson \\ Division of Solid State Physics, Lund University, Box 118, SE-221 00 Lund, Sweden
}

(Received 27 December 2000; accepted for publication 8 May 2001)

\begin{abstract}
We have studied self-assembled InAs quantum dots embedded in an InP matrix using photocapacitance and photocurrent spectroscopy. These dots are potentially promising for memories due to the large confinement energy for holes. In this work we have realized simple quantum dot memory by placing the dots in the space-charge region of a Schottky junction. Our measurements reveal that a maximum of about one hole can be stored per dot. We also find that illumination for an extended period deletes the stored charge. We show that these limitations do not reflect the intrinsic properties of the dots, but rather the sample structure in combination with deep traps present in the sample. (C) 2001 American Institute of Physics. [DOI: 10.1063/1.1382628]
\end{abstract}

During the last few years much attention has been devoted to the growth and characterization of self-assembled semiconductor quantum dots. The strong interest in these semiconductor nanostructures is motivated by the possibility to use them as active media in future high-speed electronic and photonic devices, as well as their fascinating atomic-like properties. Self-assembled InAs quantum dots embedded in an InP matrix are an interesting material system from the point of view of physics due to an unusually large confinement energy for holes of about $400 \mathrm{meV} .{ }^{1}$ Furthermore, the dots exhibit a complex electronic structure that is observed in absorption spectroscopy. ${ }^{2}$ A strong luminescence at about $1.70 \mu \mathrm{m}$ implies that the dots may be suitable for optoelectronic components compatible with the wavelength range of optical fibers.

The strong confinement of holes suggests that the dots could also be implemented in memories. Lundstrom et al. ${ }^{3}$ recently reported on exciton storage in self-assembled InAs dots in GaAs. In their approach the memory concept was based on dissociation and separate storage of the electrons and holes of optically created excitons. In Fig. 1 we show how InAs dots located in the space-charge region of a Schottky junction prepared on InP work as a simple memory device in which a light pulse is converted into an accumulation of holes in the dots, to be retrieved later by injecting electrons into the dots. The large surface dot density, together with the large confinement energy for holes, suggests that this material system is promising for room-temperature optical memory devices with high pixel (dot) density. Also, it should be possible to store enough holes in each pixel to give a reasonable gray-scale resolution. In this work we have studied properties related to the storing of holes in this type of device.

The samples were grown by metallogranic chemical vapor deposition (MOCVD) on $n^{+}$substrates. Details of the growth procedure can be found in Ref. 4 . The dots are incorporated into the middle of a $4 \mu \mathrm{m}$ not intentionally doped InP epitaxial layer. The surface density of the dots is expected to be $2 \times 10^{10} \mathrm{~cm}^{-2}$ as determined from atomic force microscope (AFM) measurements on uncapped samples. Schottky junctions were prepared by evaporating a semitransparent $100 \AA$ thick gold layer through a contact mask

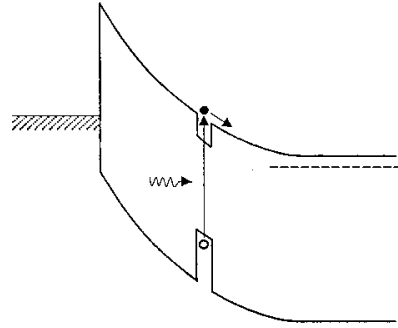

(a)

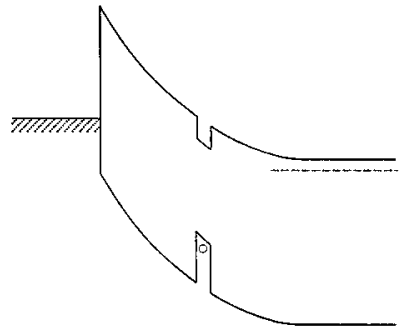

(b)

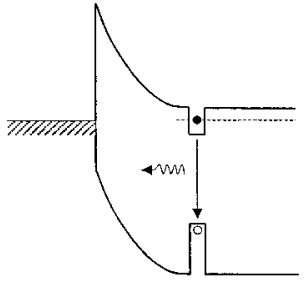

(c)

FIG. 1. Schematic description of an InAs quantum dot memory. (a) In "write" mode an incoming light pulse is converted into an accumulation of holes in the dots. The reverse bias in this mode must exceed about $1.5 \mathrm{~V}$. (b) In "store" mode the holes are stored for a very long time due to the large confinement energy for holes. (c) In "read" mode the light pulse is retrieved by injecting electrons from the bulk into the dots.

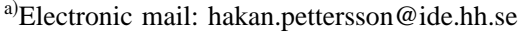




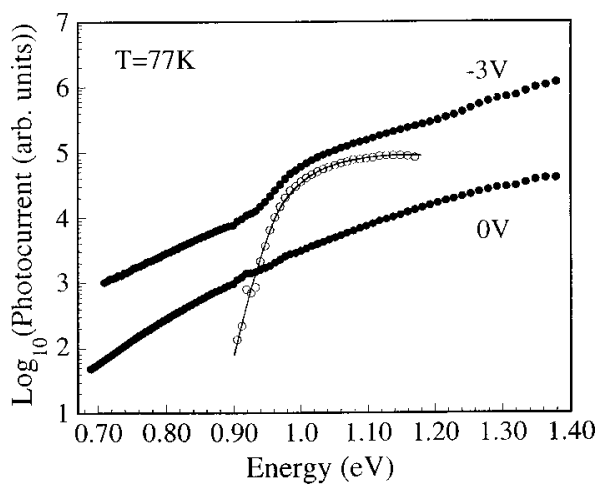

FIG. 2. Spectral distribution of the photocurrent, normalized with respect to the photon flux, measured at -3 and $0 \mathrm{~V}$ detection bias, respectively. The $-3 \mathrm{~V}$ spectrum was shifted upward for clarity. The open circles were calculated by subtracting the background from the $-3 \mathrm{~V}$ spectrum and represent current generation in the dots.

with circular apertures in about $550 \mu \mathrm{m}$ diameter. The back ohmic contact was formed by alloying $\mathrm{Au}-\mathrm{Ge}$ to the $n^{+}-\mathrm{InP}$ substrate.

The experimental methods invoked in the study were photocurrent and photocapacitance spectroscopy. These techniques have a long tradition of studying isolated point defects in bulk semiconductors, and we have previously shown that they are also excellent tools for studying quantum dots. ${ }^{1,5,6}$ In photocapacitance spectroscopy, ${ }^{7}$ a quiescent reverse bias defines the width of the space-charge region, $W$, of the Schottky junction. A short-circuit pulse contracts the space-charge region to $W_{0}$ enabling traps within $W-W_{0}$ to capture electrons from the bulk (for $n$-type material). After the filling pulse the original reverse bias is retrieved. Following this voltage cycle the sample is illuminated with monochromatic light. Changes in the occupancy of traps induced by the light results in a change in $W$ that can be monitored by measuring the differential capacitance $C$. The relationship between $C$ and $W$ is given by the expression for a plate capacitor: $C=\varepsilon \varepsilon_{0} A / W$, where $A$ is the area of the Schottky junction and $\varepsilon \varepsilon_{0}$ is the dielectric constant. In photocurrent spectroscopy ${ }^{7}$ the reverse bias is kept constant while measuring the photocurrent induced by a two-step excitation process involving traps located within $W$.

In Fig. 2 we show the spectral distribution of the photocurrent recorded at detection bias of -3 and $0 \mathrm{~V}$, respectively. As pointed out above, the dots have to be within the space-charge region in order to be detected by the experimental techniques employed in this work. $C-V$ and deep level transient spectroscopy (DLTS) measurements have shown that the space-charge region includes the dot layer for reverse biases exceeding about $1.5 \mathrm{~V} .{ }^{1}$ From this we conclude that only for the $-3 \mathrm{~V}$ spectrum are signatures of the dots to be expected. For this spectrum we observe an optical response with a threshold energy at about $0.90 \mathrm{eV}$ which we attribute to optical transitions between hole states in the dots and the conduction band of the InP barrier. Adding a binding energy of $0.45 \mathrm{eV}$ for the dots' hole ground state found in Ref. 1 results in a value of $1.35 \mathrm{eV}$ which is slightly lower than the band gap of $\mathrm{InP}(1.40 \mathrm{eV}$ at $77 \mathrm{~K})$. This discrepancy can easily be explained by noting that the optical transitions to the barrier involve the dot-related wetting layer. In Fig. 3 we see the effect of these transitions in terms of hole storage.

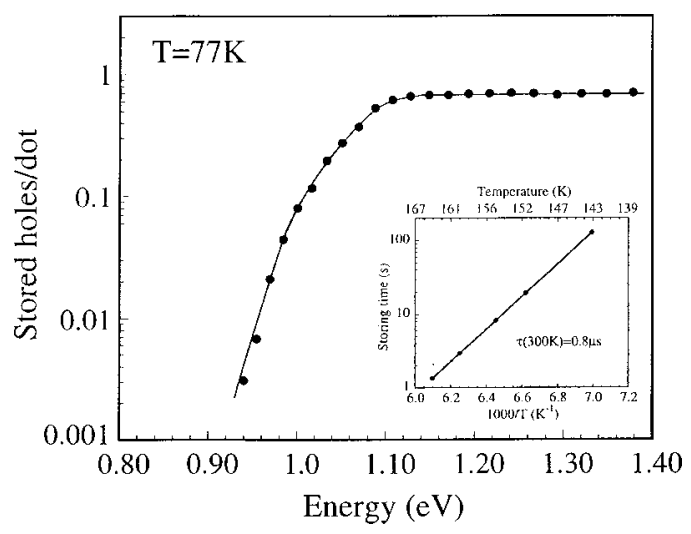

FIG. 3. Spectral dependence of stored charge in the dots. The inset shows the lifetime of stored holes at different temperatures.

Here we have plotted the spectral distribution of the photocapacitance signal, converted into an average number of stored holes/dot, detected at $-3 \mathrm{~V}$. The conversion is done as follows: The photocapacitance signal, $\Delta C$, is interpreted in terms of the total number of holes, $n$, stored in all dots using the expression

$$
\frac{\Delta C}{C}=\frac{n L}{W^{2} N_{D}},
$$

where $L$ is the distance of the dot layer from the surface and $N_{D}$ is the background $n$ doping $\left(\sim 10^{15} \mathrm{~cm}^{-3}\right)$. Since the average dot density is known approximately, the average number of holes per dot can readily be calculated. The storing time is limited by thermal emission of the holes. To emphasize that this quantum dot memory works at elevated temperatures, we show the temperature dependence of the storing time as an inset in Fig. 3. The storing time was determined by filling the dots with holes using a short optical pulse with photon energy $1.20 \mathrm{eV}$, and subsequently measuring the time constant of the capacitance transient due to thermal emission of the stored holes.

At low temperature the storing time is as long as hours which can be compared to the few seconds for the InAs/ GaAs quantum dot memory reported by Lundstrom et al. ${ }^{3}$ We did not extend our measurements to temperatures lower than about $145 \mathrm{~K}$ since the long time constant makes the measurements extremely time consuming.

An interesting effect is observed if the sample is illuminated for a longer duration. Figure 4 shows the average num-

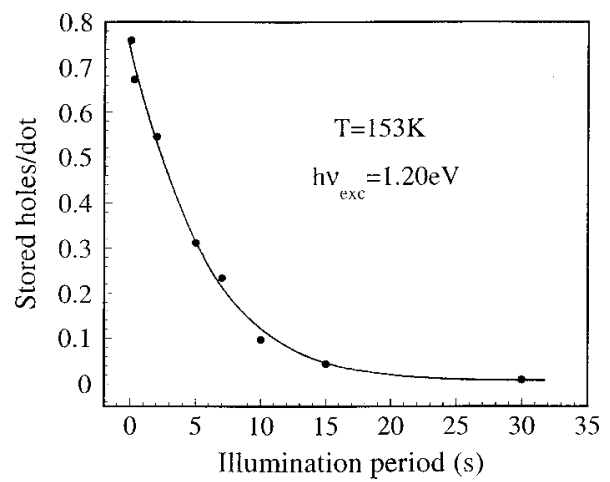

FIG. 4. Average number of holes possible to store in the dots vs the duration of illumination. 

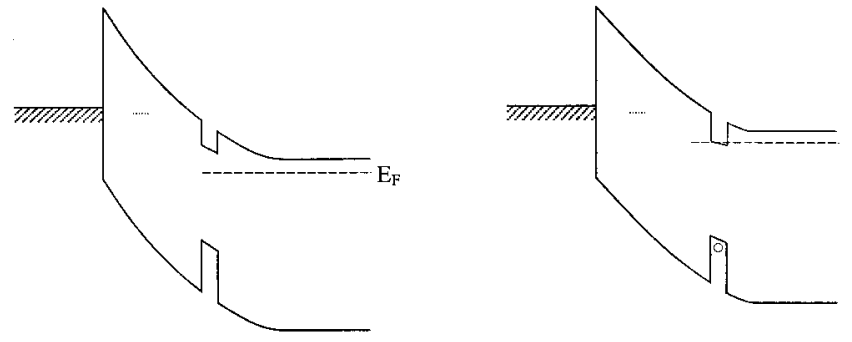

(a)

(b)

ber of holes stored per dot, determined from capacitance measurements, for different durations of illumination with $1.20 \mathrm{eV}$ photons. The sample temperature was increased from 77 to $153 \mathrm{~K}$ to facilitate convenient measurement of the number of stored holes (after illumination the holes are thermally emitted to the valence band). Evidently, the holestoring process can be completely quenched. This experimental observation offers a scheme by which not only optical filling of the dots is possible, but also optical deletion of the stored charge. As described below, we attribute this phenomenon to current generation in the dots in conjunction with the charging of traps located in the space-charge region. The light-induced loading of holes in the dots results in contraction of the width of the space-charge region, $W$, until it coincides with the position of the dot layer. At this point electrons start to diffuse from the bulk into the dots which renders further loading of holes impossible [see Fig. 5(b)]. This process limits the maximum number of holes per dot that can be stored to approximately 1 . This saturation is observed in Fig. 3 as a flat spectral distribution for photon energies exceeding about $1.10 \mathrm{eV}$. As previously discussed, the illumination leads to a photocurrent predominantly generated in the dots (Fig. 2). At steady state, holes and electrons escape from the dots at equal rates. The holes are efficiently swept away by the intrinsic electric field of the Schottky junction. On their way to the surface the holes get captured by traps. This charging process results in further contraction of $W$ which, following the argument above, leads to deletion of the stored holes in the dots [see Fig. 5(c)]. We do not dwell on the possible origin of these traps except to

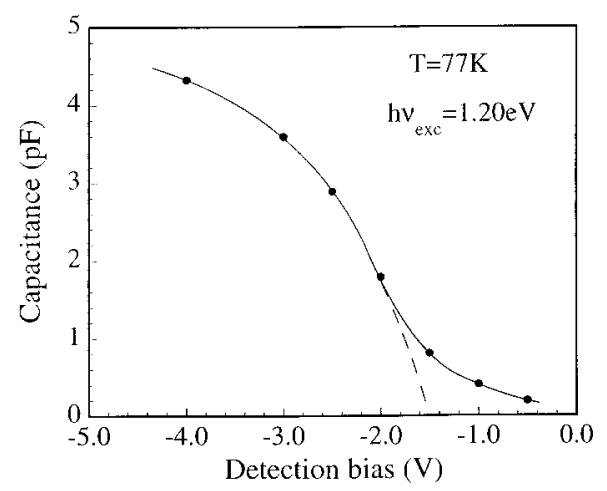

FIG. 6. Voltage dependence of the capacitance signal due to the charging of traps in the sample.

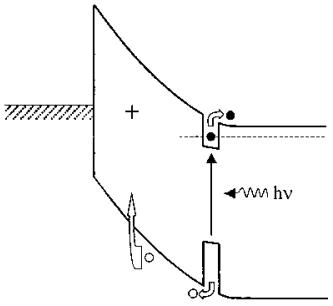

(c)
FIG. 5. Simplified energy band diagrams for the sample at a detection bias $U=-3 \mathrm{~V}$ : (a) without illumination; (b) after a short light pulse with $1.20 \mathrm{eV}$ photons inducing saturation in the stored charge; (c) continuous illumination with $1.20 \mathrm{eV}$ photons leading to quenching of the stored charge.

note that DLTS $^{8}$ measurements show that deep traps are present in the samples. Also, we found that the hole-capture process takes place relatively close to the surface since $W$ had to be decreased to less than about $1 \mu \mathrm{m}$ in order to neutralize the traps. In Fig. 6 we have plotted the voltage dependence of the capacitance change due to charging of the unknown traps. We note that there is a threshold voltage of about $-1.5 \mathrm{~V}$, which is consistent with our previous comment that the charging current predominantly originates from the dots. The increase in capacitance with increasing detection bias merely reflects the fact that $W$ increases with increasing detection bias which allows more charge to be stored in the traps before $W$ again reaches the dot layer and blocks current generation.

In conclusion, we have studied self-assembled InAs quantum dots embedded in InP using photocapacitance and photocurrent spectroscopy. The sample structure is a simple quantum dot memory device with dots located in the spacecharge region of a Schottky junction. We show that this device can store holes for about $1 \mu$ s at room temperature and for hours at $77 \mathrm{~K}$. In particular we find that the number of holes that can be stored in the dots depends on the duration of illumination. We interpret this result in terms of recombination of stored holes with electrons that diffuse from the bulk as the space-charge region of the junction contracts. This contraction stems from the charging of traps present in the sample.

The authors gratefully acknowledge financial support from the Swedish Natural Science Council, the Swedish National Board for Industrial and Technological Development, the Swedish Research Council for Engineering Sciences, and the Swedish Foundation for Strategic Research.

${ }^{1}$ H. Pettersson, C. Pryor, L. Landin, M.-E. Pistol, N. Carlsson, W. Seifert, and L. Samuelson, Phys. Rev. B 61, 4795 (2000).

${ }^{2}$ H. Pettersson, R. J. Warburton, J. P. Kotthaus, N. Carlsson, M.-E. Pistol, W. Seifert, and L. Samuelson, Phys. Rev. B 60, R11289 (1999).

${ }^{3}$ T. Lundstrom, W. Schoenfeld, H. Lee, and P. M. Petroff, Science 286, 2312 (1999)

${ }^{4}$ N. Carlsson, T. Junno, L. Montelius, M.-E. Pistol, L. Samuelson, and W. Seifert, J. Cryst. Growth 191, 347 (1998).

${ }^{5}$ S. Anand, N. Carlsson, M.-E. Pistol, L. Samuelson, W. Seifert, and L. R. Wallenberg, Appl. Phys. Lett. 67, 3016 (1995).

${ }^{6}$ H. Pettersson, S. Anand, H. G. Grimmeiss, and L. Samuelson, Phys. Rev. B 53, R10497 (1996).

${ }^{7}$ H. G. Grimmeiss and C. Ovrén, J. Phys. E 14, 1032 (1981).

${ }^{8}$ D. V. Lang, J. Appl. Phys. 45, 3014 (1974). 